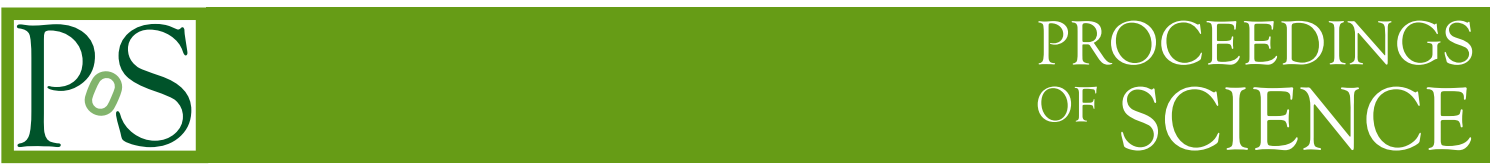

\title{
Diversity and Inclusion Activities at the Belle II Collaboration
}

\author{
Shanette A. De La Motte, ${ }^{a, *}$ Matthew Barrett ${ }^{b}$ and Elisabetta Prencipe ${ }^{c}$ \\ ${ }^{a, 1}$ Department of Physics, University of Adelaide \\ Adelaide South Australia 5005, Australia \\ ${ }^{b, 1}$ Institute of Particle and Nuclear Studies \\ High Energy Accelerator Research Organization (KEK), \\ 1-1 Oho, Tsukuba, Ibaraki, 305-0801, Japan \\ ${ }^{c, 1}$ Justus-Liebig-Universität Gießen, \\ 35392 Gießen, Germany
}

E-mail: shanette.delamotte@adelaide.edu.au

The Belle II collaboration has over 1000 people from institutions in 26 countries working together to achieve its physics goals. The collaboration is committed to fostering an open, diverse, and inclusive environment. In order to do so, Belle II created a diversity office to raise awareness of diversity and inclusion issues; promote an inclusive atmosphere within the collaboration; provide a safe and confidential point to contact for collaborators to report any issues, particularly those related to discrimination and harassment; and ensure that persons from underrepresented groups are considered for positions of responsibility within the collaboration.

These proceedings accompany the talk delivered during EPS-HEP 2021 [1]. Diversity and inclusion activities and initiatives at Belle II will be discussed and an analysis of the evolving demographics of the collaboration is presented.

*** The European Physical Society Conference on High Energy Physics (EPS-HEP2021), ***

*** 26-30 July $2021 * * *$

*** Online conference, jointly organized by Universität Hamburg and the research center DESY ***

\footnotetext{
${ }^{1}$ On behalf of the Belle II Collaboration

${ }^{*}$ Speaker
} 


\section{Belle II, Diversity and Inclusion, and the importance of gathering data}

The Belle II collaboration is a collection of high-energy physics researchers who aim to investigate the properties of $b$-quarks at the luminosity frontier. These investigations are carried out using the the Belle II detector, located at the SuperKEKB electron-positron collider in Tsukuba, Japan. Most recently, Belle II surpassed both the original Belle and Babar experiments in achieving the world record for integrated luminosity recorded in a single month, in May 2021. Not only is it an aim of Belle II to continue breaking records, but also to be an inclusive collaboration that allows all peoples to do particle physics.

Diversity and Inclusion are terms that need to be defined carefully, so as to fully understand their importance within a scientific collaboration. Diversity describes the large range of differences between people. Examples can include (but are not limited to) differences in gender, race, sexual orientation, ability, or socio-economic status. A quick assessment of one's own working groups will most likely indicate that the gender and racial diversity of wider society isn't always represented at scientific institutions, let alone in positions of leadership at these institutions. Thus, inclusion describes actions we can take to support those who may not be seen as often, to give them an equal opportunity to do good physics.

The gathering of data is essential for ensuring inclusion measures are appropriate for a given collaboration, as well as discovering new areas that may require more attention. According to Nature Reviews: Physics[2], "with inadequate data collection and analysis, progress towards equal representation remains slow", suggesting that obtaining high-quality demographic data is the first step in creating an inclusive workplace. In this way, data collection can reveal unknown discrepancies and thus leadership can implement changes to rectify this. One such example from Belle II, where collecting data led to new understanding, as in a 2018 membership survey. Members were invited to share their experiences within the collaboration, inspired by a similar survey held by LHCb. Over 6 months 240 collaborators responded, a $1 / 4$ of all members at the time. From this survey, it was found that around $25 \%$ of the respondents had, at some point in their career, "withdrawn from consideration for a leadership role at Belle II (implicitly or explicitly) because of the impact it would have on [their] family life." This emphasised the need in our collaboration to be more respectful of work-life balance.

\section{The Demographics of Belle II Collaborators}

Belle II collaborators come from institutions spread across 4 continents, with $37 \%$ of our members residing in Asia.

Figure 1a is a bar chart summarising the genders of Belle II collaborators in 2020, broken down by region of their institute. Regional grouping, as opposed to individual countries, is used to ensure anonymity of our members from smaller institutes. This particular grouping allowed for comparison with a similar study by the North-American-dominated ATLAS Experiment in 2016 [3]. Contrasting with ATLAS, the fraction of women in each of the regions of Belle II as of March 2021, varies from 9.7\% in America to $21.9 \%$ in Asia. A higher fraction of women in regions geographically closer to the experiment is seen - this could be due to a combination of factors, so it is difficult to further qualify why this is the case. 


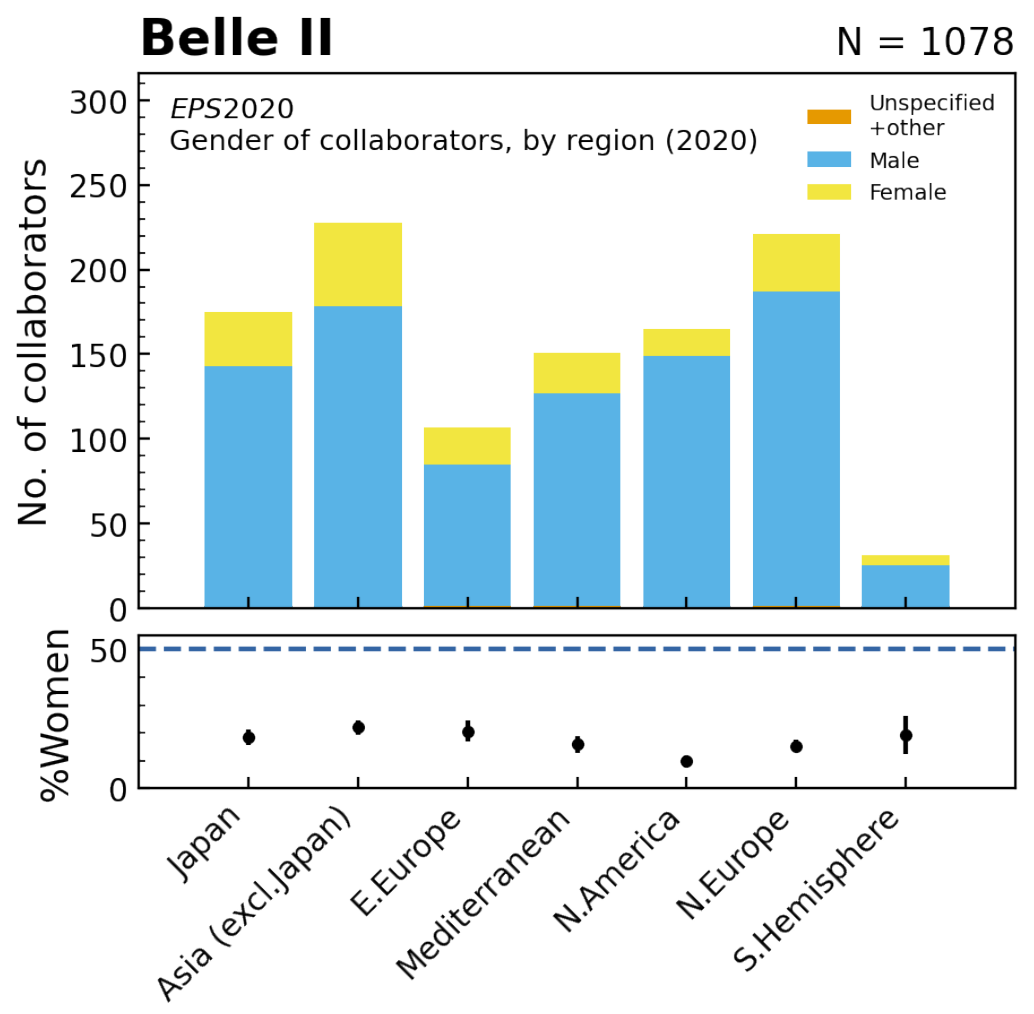

(a)

\section{Belle II}

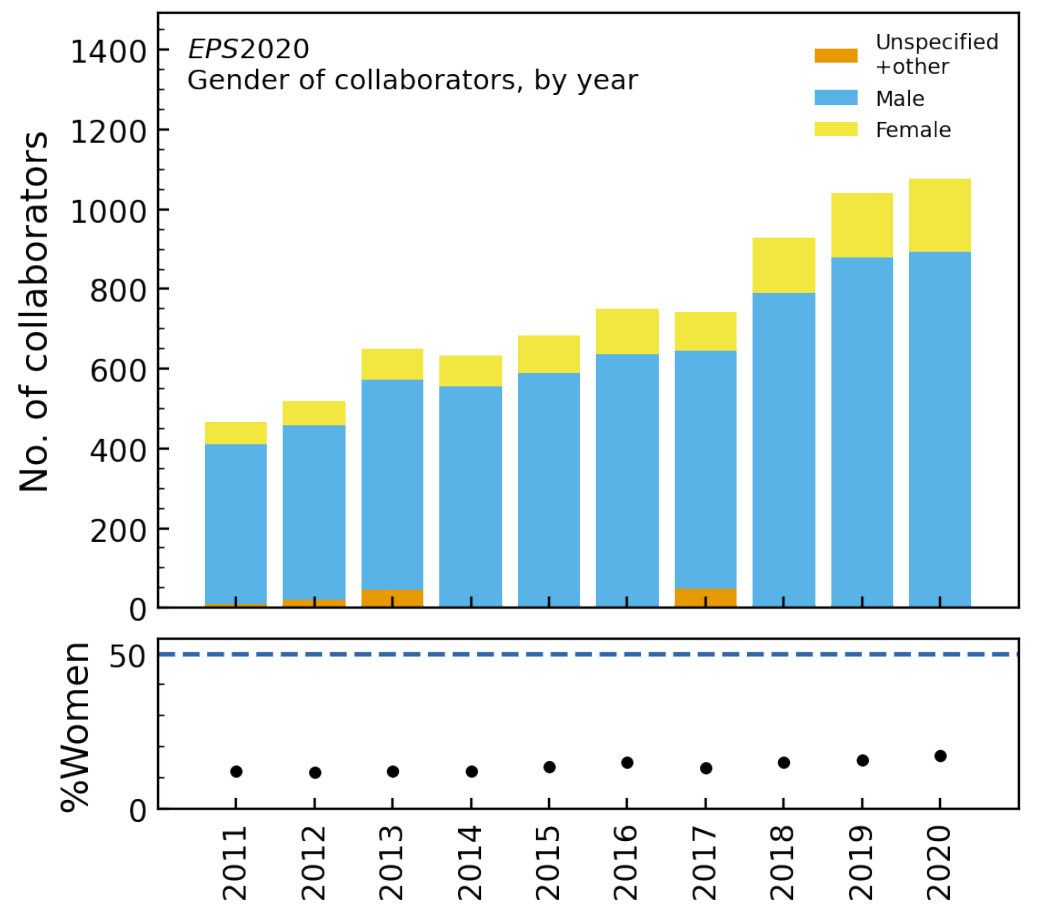

(b)

Figure 1: Belle II demographics: Gender of collaborators, broken down by region and by year 
Figure $1 \mathrm{~b}$ also summarises the genders of Belle II, in this case showing the growth of our collaboration from 2011. This marks marks the year when Belle II membership became independent from the original Belle experiment. The gender gap in Physics is one of the highest in science and Belle II is sadly no different. While our number of collaborators has almost doubled, our fraction of women has not increased at the same rate, going from $12 \%$ to $17 \%$. If we naively extrapolate this trend, it would take 60 years to reach parity, beyond the lifetime of the experiment.

Data for these plots are taken from the Belle II internal membership system, where users are offered the ability to declare their gender as male, female or other. Other is implemented in order to offer an option to those who identify as non-binary, though the bar chart demonstrates this not a frequently selected option. Some collaborators have chosen to leave their gender unspecified in their enrolment at Belle II. Steps are being taken to give users the ability to choose their pronouns during their enrolment, so that others may be more sensitive in their use of language.

It's important to acknowledge the limitations of collecting demographic data in this way. Declaring one's gender during their enrolment may pose some challenges to queer collaborators, whose identity might not be known publicly or professionally.

Figure 2a breaks down the genders of Belle II collaboration based on their academic positions. A larger fraction of women at postgraduate level is seen, becoming much smaller at professional level. Thus, the percentage of collaborators that are women drops as their career progresses from postgraduate to permanent faculty in a statistically significant way. This lends evidence that Belle II is not exempt from the wider "leaky pipeline" effect observed in science, that there are less women with each higher stage of academia.

Figure $2 \mathrm{~b}$ demonstrates the yearly involvement of women in the Belle II collaboration. Here, the fraction of women overall in a given year can be compared to the fraction of women chosen for external conference talks, the fraction offering internal conference talks at our Belle II General Meeting and the fraction appointed to any leadership roles and as well as in a senior role. We roughly see the participation of women grow towards that of the overall collaboration, though we must acknowledge the choppiness in the trends due to the small number of women at each level.

\section{Inclusion efforts at Belle II}

Having quantified some of the issues within the Belle II demographics, the inclusion efforts implemented to support minorities in gender, race and sexuality will be discussed. In this way Belle II can combat the trends seen in the previous section.

In October 2017 the Belle II code of conduct was amended to explicitly include that "members shall not engage in violent, harassing, sexist, racist, or discriminatory behaviour" [4]. The code of conduct is to be followed by all members who sign on to Belle II. Most importantly, it acknowledges "that an array of values, interests, experiences and cultural viewpoints enriches our learning".

A year later in 2018, Belle II elected two diversity officers. Their roles include; promoting an inclusive collaboration; to provide a safe and confidential point of contact to report issues, particularly those related to discrimination, bullying, or harassment; to support those from marginalised groups and ensure they are appropriately considered for positions of responsibility and; to encourage and publicize the collaboration's events and efforts promoting equity. 


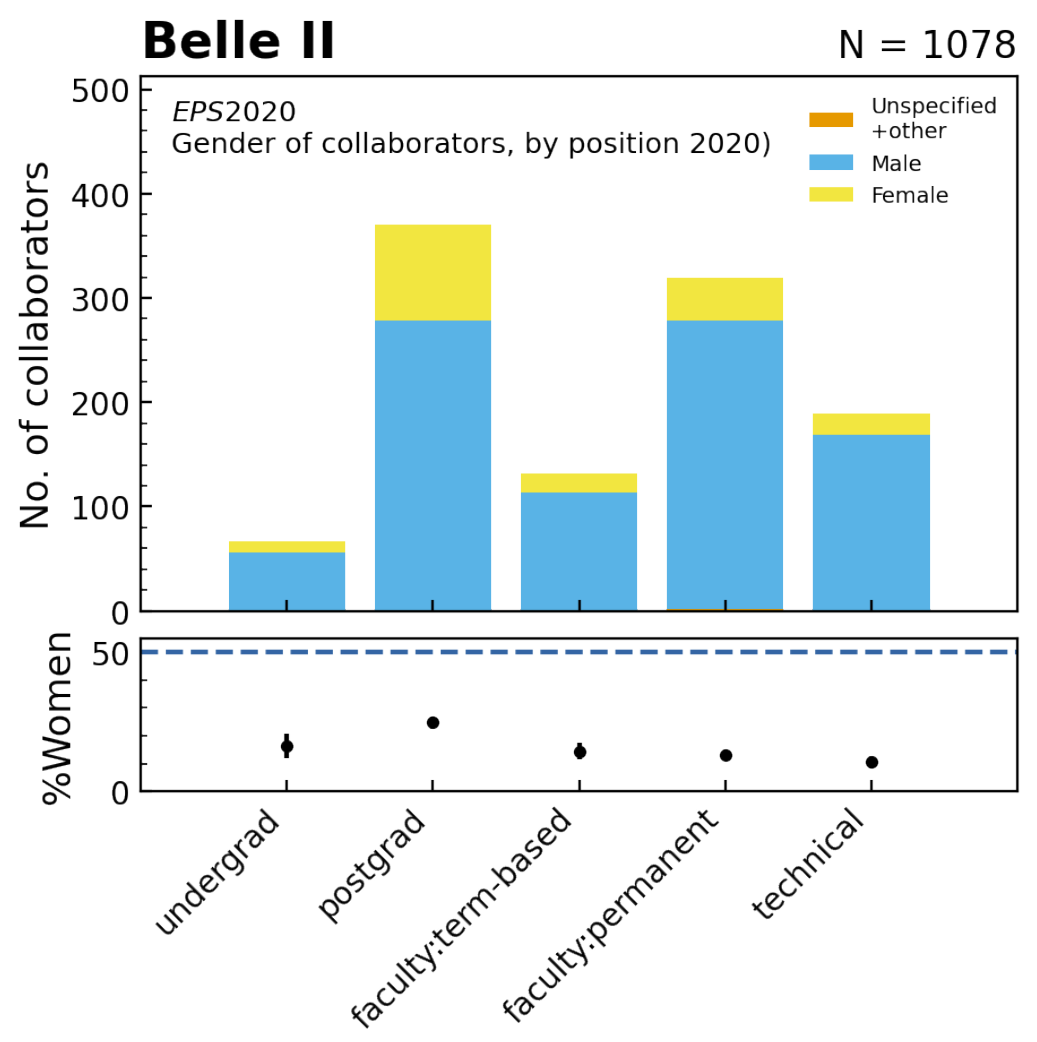

(a)

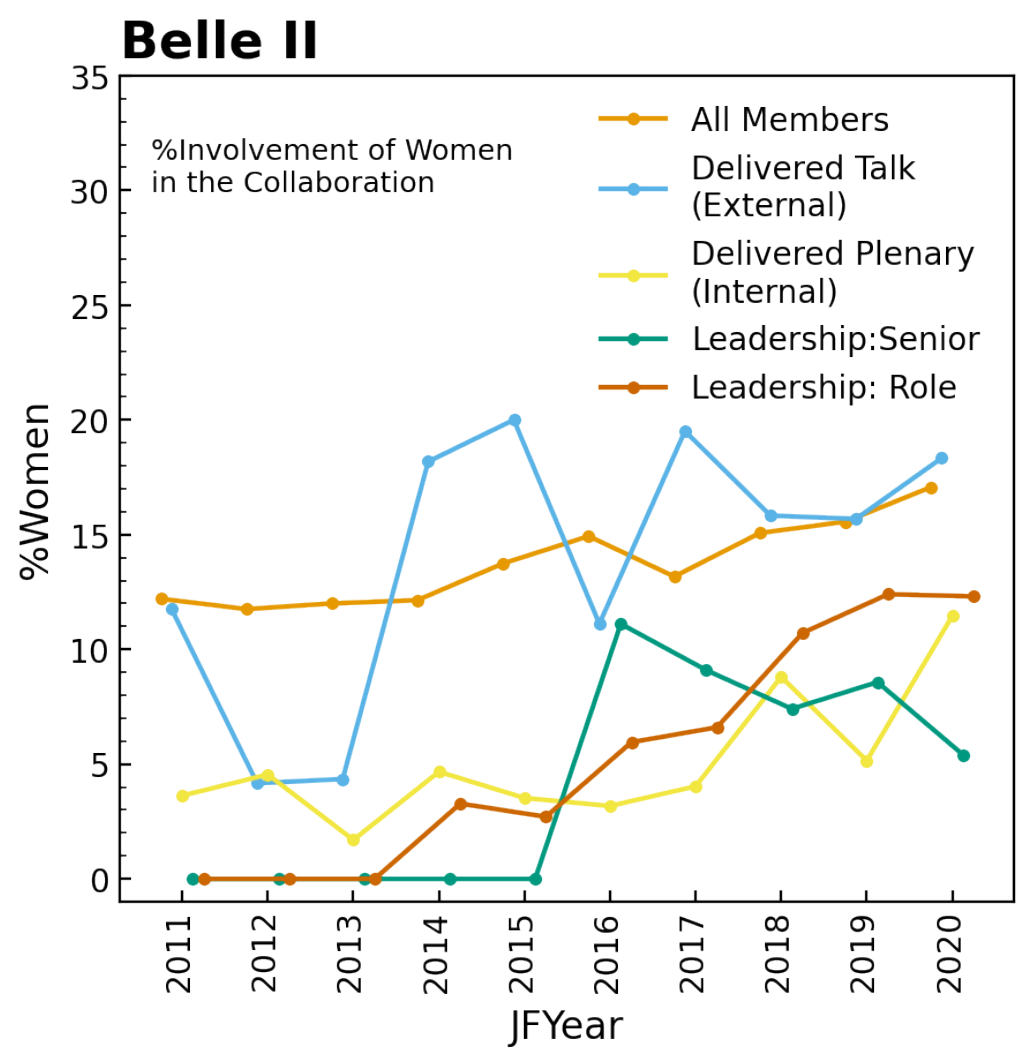

(b)

Figure 2: Belle II demographics(cont.): Gender of coflaborators by position and percentage participation of women within the collaboration 
External to the collaboration, Belle II is active on Facebook, Twitter, and Instagram. Social media is used not only to publicise scientific achievements, but also to bring attention to events such as International Women's Day, International Day of Women and Girls in Science, LGBTQ+STEM Day and Colo(u)r Blind Awareness Day. To that effect, analysts are encouraged to use color schemes accessible to those colour vision deficiency[6], so that Belle II results may be interpretable by all. Belle II would like to extend its social media presence to include profiles of collaborators, to inspire more scientists from underrepresented areas to join the collaboration.

Belle II became an official backer of LGBTQ+STEM Day[7] in 2019, thus pledging to make all members aware of the collaborations support for the queer community. Belle II joins other high energy physics experiments, such as ATLAS and LHCb, as supporters for LGBTQ+STEM Day, though it remains the only group outside of the US and Europe. To help the organisers of LGBTQ+STEM to publicise efforts outside of the western community, in particular to other Japanese institutes, Belle II queer support posts are in both Japanese and English. Unfortunately, publicly supporting diversity in sexuality has provided some challenges. The Belle II pride display picture was once used in a social media post with an unrelated photograph of members, where one member originated from a country with strict punishments for supporting the queer community. With such a internationally diverse collaboration, it's important to be sensitive to the diversity in cultures and political opinions brought to Belle II. However, Belle II will continue to be allies to queer collaborators. Checks have now been strengthened to ensure proper permissions are obtained from those photographed before publicising via the Belle II social media.

Belle II has also made efforts to make it's language more inclusive. Following similar changes within the git community [8], the computing and software groups have removed the use of the word "slave" within the Belle II Analysis Framework. The term is implemented in computing to describe a system which follows another, but has deep underlying racial connatations in it's use. In some cases, the changing of terminology is not as straightforward, such as when the word is present due to external software requirements. ROOT is a data framework created by CERN that is indispensible for large data analyses, but contains functions like "SlaveTerminate". Belle II would like to work with the ROOT developers to rectify this issue. Similarly the term for the followed system, "master", has also been removed from Belle II's code repositories. There is some resistance as this term is much more common in computing. Alternatives include using the term "main", for things such as a primary development branch in git, as has been performed in the now publically available Belle II Analysis Framework[9]. This is something can be easily performed in personal git repos, where git branch - $m$ allows you to effectively rename your branch. Examples of other language substitutions are to use primary and secondary instead of slave and master, as well as denylist/allowlist instead of blacklist/whitelist. These naming conventions are now available for analysts as part of the Belle II coding guidelines.

$\mathrm{KEK}$, the laboratory at which Belle II is located, is very receptive to suggested changes towards better inclusion. The control room at the site of the experiment has monitors with high contrast colour schemes, allowing those with differing kinds of colour vision deficiency to complete necessary control room operations. Another important example is the improvements on bathroom accessibility at KEK, where the onsite dormitories now include more bathrooms for women. Furthermore, a bathroom close to the control room, which previously only had urinals, has now been made both gender neutral and accessible for persons with disabilities. This required considerable 
plumbing and construction efforts as it was three floors underground. Previously, those who couldn't use the urinals had to ascend three floors to use the bathroom when on a control room shift.

As of 2021, accessing KEK has been difficult due to the Coronavirus pandemic. Control room shifts to take data at the Belle II experiment were often filled by visiting members, meaning luminosity targets were at risk. Plans were rapidly changed for the 2020 and 2021 Physics runs, where Belle II relied on local members to take on additional control room and subdetector expert shifts. Despite these changes, Belle II managed to actually break the worlds' instantaneous luminosity record in June 2020. Another way in which Belle II was affected by the Coranavirus pandemic was in it's collaboration-wide meetings. Usually held 3 times a year, these were moved from taking place at KEK to becoming online-only. The only other time this happened was in 2011 after the Great East Japan Earthquake. With the collaboration split between multiple timezones, holding meetings purely online can result in collaborators balancing their daily work in their home timezone and attending the meetings in another completely different one. These meetings at inconvenient hours may be more of a burden for those with dependents. In an attempt to ameliorate this, meeting sessions were recorded and sessions are replayed at multiple times. Some meetings are split into multiple sessions to allow presenters to choose a friendlier time slot. When international travel becomes an option again, collaborations will need to reflect on whether to keep some or all of their meetings remote. A totally remote conference setup will improve accessibility, for those who may have family commitments or are from underrepresented regions. However, there is immense value in in-person networking experience, which simply can't be reproduced via online video.

Another issue of interest to the Belle II Diversity Office is addressing childcare for visitors to KEK. Finding childcare can be challenging for visitors at KEK, especially for those who may not speak Japanese. This can discourage caregivers (a role which disproportionately affects women) from pursuing research opportunities on campus. KEK has partially subsidized the cost of childcare, though currently this has only been available for KEK staff. The Belle II secretariate has assisted in finding childcare for international visitors, though more effort must be made to look into options that can better accommodate and publicise these possibilities.

\section{Conclusion}

Overall, Belle II would like to continue raising awareness of diversity and inclusion. This is not only to be a better collaboration for members, but to make high energy physics a more inclusive space, and better the field of Physics as a whole. The Belle II diversity officers are open to suggestions and ideas, as there is still a lot more effort needed to ensure the diversity of the wider population is reflected in physics collaborations. It is through support for underrepresented groups that Belle II can obtain more diverse approaches to analysis and thus better physics.

\section{References}

[1] This conference note is accompanied by the slides delivered during the talk, available at https://indico.desy.de/event/28202/contributions/106206/attachments/ 67635/84395/Diversity\%20and\%20inclusion\%20activities\%20at\%20the\% 20Belle\%20II.pdf. A companion script to the talk is also available at https: 
//indico.desy.de/event/28202/contributions/106206/attachments/67635/ 84397/CompanionScript_DiversityAndInclusionActivitiesAtBelleII .pdf

[2] Nature Reviews: Physics, https://www . nature.com/articles/s42254-019-0061-3

[3] Studies related to gender and geographic diversity in the ATLAS Collaboration, ATLAS Collaboration https://cds. cern. ch/record/2202392/files/ATL-GEN-PUB-2016-001. pdf

https://atlas.web.cern.ch/Atlas/GROUPS/PHYSICS/PUBNOTES/

ATL-GEN-PUB-2016-001/fig_03.png

[4] Belle II Diversity Statement <https://belle2 . jp/diversity/>

[5] Belle II can be found on Facebook at https://www. facebook.com/belle2collab (English) https: / /www . facebook. com/belle2 japan (Japanese), Twitter (English) at https: //twitter.com/belle2collab?lang=en, Twitter (Japanese) https://twitter.com/ belle2japan and Instagram https://www . instagram.com/belle2collab/?hl=en

[6] Color Universal Design (CUD) - How to make figures and presentations that are friendly to Colorblind people https://jfly. uni-koeln.de/color/

[7] https://prideinstem.org/lgbtstemday/

[8] Software Freedom Conservancy, published 23/06/2020, Regarding Git and Branch Naming, accessed 26/10/2020 <https://sfconservancy.org/news/2020/jun/23/ gitbranchname/>

[9] https://github.com/belle2/basf2 Belle II Analysis Framework

\section{Acknowledgements}

The authors would like to thank the Belle II Secretariat, Belle II Collaborative Services, and the Belle II Speakers Committee for their invaluable assistance and for maintaining Belle II membership and conference statistics. Shanette, as a PhD student of the University of Adelaide in Australia, would like to acknowledge the Traditional Owners and Custodians of the lands that she lives and works on. She pays her respects to the Kaurna people and to Indigenous Elders past, present and emerging. Sovereignty has never been ceded. It always was and always will be, Aboriginal land. 